\title{
Human inspired effort distribution during collision avoidance in human-robot motion
}

\author{
Grimaldo Silva ${ }^{1}$, Anne-Hélène Olivier ${ }^{2,3}$, Armel Crétual ${ }^{2,3}$, \\ Julien Pettré ${ }^{3}$ and Thierry Fraichard ${ }^{1}$
}

\begin{abstract}
Recent works in the area of human robot motion showed that behaving in a human-like manner allows a robot to reduce global cognitive effort for people in the environment. Given that collision avoidance situations between people are solved cooperatively, this work models the manner in which this cooperation is done so that a robot can replicate their behavior. To that end, hundreds of situations where two walkers have crossing trajectories were analyzed. Based on these human trajectories involving a collision avoidance task, we determined how total effort is shared between each walker depending on several factors of the interaction such as crossing angle, time to collision and speed. To validate our approach, a proof of concept is integrated into ROS with Reciprocal Velocity Objects (RVO) in order to distribute collision avoidance effort in a human-like way.
\end{abstract}

\section{INTRODUCTION}

Consider a robot whose goal is to reach a particular target. In between its current position and its goal, this robot can encounter an unbounded number of static and dynamic obstacles. Our focus in this work lies on the interaction behavior when avoiding dynamic obstacles, specifically people. We are interested in studying how a robot should move among people, what we define as a Human Robot Motion (HRM) problem. In the context of HRM, it is not sufficient to only guarantee collision-free motions [1]. The main objective is to provide robots with human-like behavior when avoiding collision with people, which can involve a broad range of concepts such as social spaces [2] and legibility concerns [3], in what we call an appropriate motion.

In the present work, we focus on one characteristic of human-human interaction during collision avoidance that is the mutual sharing of the adaptations performed to solve a collision [4]. We aim at better understanding the contribution of each walker to avoid a collision, which is expressed in terms of the amount of change in both desired energy and time to the goal, what we call collision avoidance effort. We hypothesize that replicating this human behavior in a robot would imply that people will be able to rely on their implicit

*This work is partially supported by the Brazilian National Counsel of Technological and Scientific Development (CNPq) and the LabEx PERSYVAL-Lab (ANR-11-LABX-0025-01) funded by the French program Investissement d'avenir.

${ }^{1}$ Univ. Grenoble Alpes, Inria, CNRS, Grenoble INP, LIG, 38000 Grenoble, France

2 Lab. Mouvement Sport Santeé (M2S), UFRAPS, Université Rennes 2-ENS Cachan, Avenue Charles Tillon, CS24414, 35044 Rennes, France

${ }^{3}$ INRIA, Centre de Rennes Bretagne Atlantique, Campus Universitaire de Beaulieu, 263 avenue du Général Leclerc, 35042 Rennes, France

E-mail: jose.jgrimaldo@gmail.com, armel.cretual@univ-rennes2.fr, $\{$ anne-helene.olivier, julien.pettre, thierry.fraichard\}@inria.fr natural behavior when avoiding a collision with a robot [5]. This could be an advantage given that human-like motion has been shown to reduce planning effort for all the people in the environment [6]. In this context it is also worth noting that people tend to choose collision avoidance strategies based on situational factors, such as heading, rather than personal characteristics [7] such as gender or personality - or lack thereof in case of a robot.

Recent works have studied the proportional contribution to avoid a collision in perpendicular crossing scenarios between two walkers [4]. They found that there is a statistically significant difference in the amount of contribution performed by each walker to reduce the perceived risk of future collision. This contribution is done by adapting their respective trajectories depending on their role in the collision avoidance: the walker who arrives second at the crossing performed more adaptation than the walker who arrives first. Authors explained this as a consequence of the asymmetric shape of personal space. To further expand these results for a wider range crossing angles (not just perpendicular) and also to better characterize this behavior, our work presents a model designed from the evaluation of hundreds of collision avoidance situations between two walkers.

Our approach is composed of two core components that are a result of this evaluation. The first component is a definition of collision avoidance effort, while the other component is a representation of the effort distribution based on a number of situational factors that describe a given collision situation. These components are tested using the Robot Operating System (ROS) with Reciprocal Velocity Objects (RVO) [8] to simulate the navigation of both people and robot. However, instead of RVO's standard equal share, our approach is used to predict the amount of contribution to avoid a collision that is expected from each agent.

To our knowledge, this is the first work that explicitly accounts, based on empirical data from walkers in collision avoidance situations, for the manner in which a person expects the effort to be shared in a given scenario.

\section{A. Outline of the paper}

In Sec. II, the state-of-the-art is presented and previous works are contrasted with our method. Our first contribution, a measure of collision avoidance effort for a given trajectory, is presented in Sec. III. This measure and the manner in which people distribute it in a given collision situation are then modeled based on the evaluation of hundreds of collision avoidance scenarios in Sec. IV. To evaluate the 
impact of these changes in actual trajectories, simulated experiments are done into ROS using RVO in Sec. V. Finally, the conclusion is presented in Sec. VI.

\section{State-of-the-art and contributions}

Understanding and replicating human behavior has been a common trend in recent human-robot motion research. To that effect, many human-like models have been used to allow a robot to replicate or account for a person's behavior. For example, in [9], a robot is able to predict how standing people that are inadvertently blocking its way will react to its motion, that is, in which manner they will allow the robot to pass and how much effort this requires. This allows a robot to pass through regions that would have been hard or impossible otherwise. This is done through the use of the Social Force Model (SFM) [10] to calculate people's reaction (as a group or individual) to a particular robot plan.

For situations when people are already in motion, in [11] a robot navigated inside a shopping mall using an approach also based on SFM. However, in their work, the amount of contribution that each person did to avoid a collision to the robot was not compared to a people-only baseline. To better quantify the amount of change in a person's motion caused by the robot, in [12], a measure, called social work, was used as an additional cost for each candidate trajectory to the robot's goal. Such formulation allows one to give precedence to trajectories that have smaller impact on the motion plan of nearby people.

In the aforementioned works, the impact of a robot trajectory in a person (or group) is minimized without accounting for people's expectations. However, in [4] it was shown that humans walkers mutually solved the collision avoidance task but their contribution is not symmetric. In particular, in an orthogonal crossing situation, a person who will arrive second at the crossing contributes more than the other to avoid the collision in terms of motion adaptation. Therefore, attempting to solve a collision situation by simply minimizing the impact in a person's original motion plan would lead to a robot behavior that is not human-like. In our work the expectations of people concerning their contribution to avoid a collision in a given situation are defined in the context of the current collision scenario, accounting for situational factors such as: crossing angle, crossing order and time to collision.

In our previous work, presented in [13], the definition of effort was based on progression to the goal i.e. the faster one arrives at the goal the better. This implies, for most cases, that one preferred speed should be its maximum speed. This is not sufficient to properly explain human behavior, as people do not walk at their maximum speed and furthermore often accelerate (or decelerate and change heading) [4] to avoid a collision. Moreover, the effort distribution in [13] does not account for several situational factors such as crossing angle and time to collision. We considered these factors because they may have an influence on the perceived future risk of collision for a given situation. Obviously, on one hand, time to collision indicates how soon the collision will occur. On the other hand, the crossing angle may change how the future distance of closest approach is projected in the personal space of the walker. Indeed, personal space was shown to be elliptical during human locomotion [14], this can change the threshold in which a person perceives their personal as being invaded when crossing through different angles. These factors (among others) and their impact are accounted for in this work, which is based on empirical results obtained from people in collision avoidance situations.

Our work brings novel contributions in the area of humanrobot motion. We present a definition of collision avoidance effort in order to describe scenarios in terms of total effort and also its distribution among people. Furthermore, we also present this as a human-like model for robots that explicitly accounts for situational factors such as crossing angle, crossing order and time to collision. This allows one to define what are the situations in which more effort is expected and also how changes in one or more factors affect the effort distribution.

\section{Effort-based trajectory cost function}

In this section, we describe the cost of a given trajectory in terms of energy and time. This cost is used to calculate the collision avoidance effort which allows one to estimate the relative effort performed by each agent in a collision avoidance interaction.

A person walking towards his/her goal dedicates a certain amount of energy to reach it, the amount of energy dedicated, for his/her chosen speed, tends to be optimal [15]. Furthermore, the total energy required for a given trajectory $\pi_{p}=\left\{\vec{p}_{p}(0), \vec{p}_{p}(1), \cdots, \vec{p}_{p}(t), \cdots, \vec{p}_{p}(n)\right\}$ with each position $\vec{p}(t)=\left(x_{p}(t), y_{p}(t)\right) \in \mathbb{R} \times \mathbb{R}$, is defined in the context of our work, based on [15], as

$$
E\left(\pi_{p}\right)=\sum_{t=1}^{n}\left(32+0.0050 v_{p}(t)^{2}\right)
$$

which represents a quadratic cost function in terms of speed $v_{p}(t)$ at time $t$ for the trajectory $\pi_{p}$ in meters per minute. It returns the cost in terms of calories per minute per kilogram.

\section{A. Describing trajectories in terms of time and energy}

A person going towards its goal without any obstacle, dynamic or otherwise, might choose what is defined as an optimal-speed walk [15] which refers to the most energy efficient (in terms of calories) walking speed for a given person. Empirical evidence found that this value for an average person is of $80 \mathrm{~m} / \mathrm{min}$ or $1.33 \mathrm{~m} / \mathrm{s}$ [15]. For a robot, finding the most human-like trajectory, at least in terms of energy efficiency, to a given goal could be defined simply as

$$
\pi_{\pi}^{*}=\underset{\pi_{r} \in \Pi_{r}}{\arg \min } E\left(\pi_{r}\right)
$$

where $\Pi_{r}$ is the set of admissible trajectories of robot $r$ to its goal - trajectories that are both safe (collision-free) and appropriate.

Outside of the optimal-speed walk, in terms of energy spent per meter walked, there are always two speeds that 
spend the same total energy: one faster than optimal-speed walking and one slower. Walking faster means smaller time to goal while walking slower can allow for greater safety, exploration and also etiquette. In this sense, at least for the task of goal reaching without obstacles there is no incentive to walking slower than the optimal-speed walk.

In the case where dynamic obstacles with potential collision risk are involved, more specifically people, one can increase or reduce their speed and also change their heading to avoid a collision. This in turn can change their original balance between the amount of energy invested and the time to the goal. This trade-off is important in the sense that it can allow even for an energy inefficient action to be justified as it helps minimize global energy cost to avoid a collision. This time and energy trade-off for a person can be represented as a trajectory cost function $\Gamma:\left[\mathbb{R}^{2}, \cdots, \mathbb{R}^{2}\right] \rightarrow \mathbb{R}$ and represents the cost of a trajectory (with fixed time step), defined as

$$
\Gamma\left(\pi_{p}\right)=E\left(\pi_{p}\right)+\zeta_{p}\left(v_{p}^{\text {des }}\right) \cdot T\left(\pi_{p}\right)
$$

where $T\left(\pi_{p}\right)$ is the time required to execute this trajectory and $v_{p}^{d e s}$ is the desired speed choice for a person, which is not necessarily the most energy efficient, in case there are no obstacles to its goal. Moreover, $\zeta: \mathbb{R} \rightarrow \mathbb{R}$ is a function of a given speed that returns a value which makes a given time energy trade-off optimal only when the agent $p$ arrives at the goal with the desired energy and time. That is, even in cases where the agent arrives earlier to the goal while using more energy or when it arrives later while using less energy, the value of $\Gamma\left(\pi_{p}\right)$ will not be minimum. Thus, for a given $v_{p}^{\text {des }}$ the value of $\zeta\left(v_{p}^{\text {des }}\right)$ that makes this chosen time energy trade-off optimal for Eq. 3 can be pre-calculated for any speed. The shape of the time energy trade-off function, given $\zeta$, is showcased in Fig. 1 .

The value $\zeta_{p}\left(v_{p}^{\text {des }}\right)$ is of fundamental importance for our formulation given that it defines the willingness of a person to spend energy to reach their goal sooner. For instance, if $\zeta$ is defined as zero, the agent will prefer the most costefficient speed. On the other hand, higher values of $\zeta\left(v_{p}^{\text {des }}\right)$ progressively indicate to the agent that higher energy costs are worth, up to a point, in order to arrive sooner to his goal.

Each person has a different trajectory energy cost function and thus a different $\zeta\left(v_{p}^{d e s}\right)$. Several ways are being considered to approximate an individual trajectory energy cost, such as using height and gender as predictors, but in the current iteration of our formulation, the most energyefficient speed is set as a grand average found in empirical tests in the literature [15].

\section{B. Collision avoidance effort}

As we established in Eq. 3, to reach a goal while avoiding a collision an agent is forced to change its time energy tradeoff to non-optimal values. This change is what we define as collision avoidance effort. Let $\Delta E\left(\pi_{p}\right)$ and $\Delta T\left(\pi_{p}\right)$ represent, respectively, the change in energy and time between the current $\Gamma\left(\pi_{p}\right)$ and the optimal time energy trade-off from an ideal situation without obstacles - the baseline. Thus, we represent collision avoidance effort as

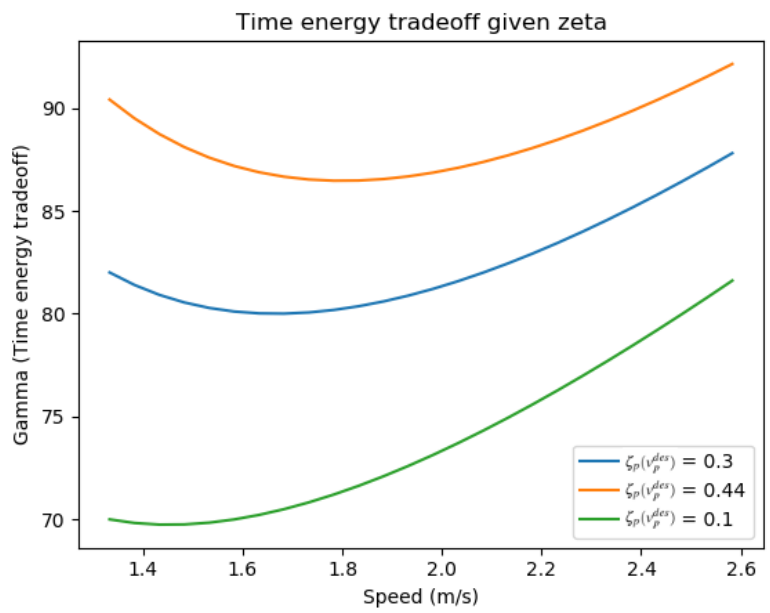

Fig. 1: Change in the time energy trade-off function in accordance to change in $\zeta_{p}\left(v_{p}^{\text {des }}\right)$ in case one walks straight to the goal without obstacles. Thus, for each case the minimal value is reached only when the agent arrives at his goal at the desired time using the desired amount of energy. Thus, in case a person accelerates, decelerates or move its heading away from the goal in order to avoid a collision its value will not ever be minimal.

$$
F\left(\pi_{p}\right)=\Delta \Gamma\left(\pi_{p}\right)=\Delta E\left(\pi_{p}\right)+\zeta\left(v_{p}^{\text {des }}\right) \cdot \Delta T\left(\pi_{p}\right)
$$

The value $F\left(\pi_{p}\right)$ will only be minimal when the person arrives in his goal with both the desired time and energy. In this case, $F\left(\pi_{p}\right)$ will be zero.

Given the aforementioned formulation, the effort distribution coefficient (EDC) of a given agent $p$, that is, their contribution to collision avoidance in relation to another agent $q$, represented as $\alpha_{p, q}$, can be directly calculated using

$$
\alpha_{p, q}=\frac{F\left(\pi_{p}\right)}{F\left(\pi_{p}\right)+F\left(\pi_{q}\right)}
$$

\section{Human-like effort distribution}

For a robot, when avoiding collision with a person it is necessary to account for social expectations instead of an approach based on, for instance, the minimization of total effort to avoid a collision. For example, a person that is coming from behind another one generally does not expect the person in front to collaborate in order avoid the collision, even if they are aware of each other. Moreover, depending on the crossing order, relative speed and relative position between people the distribution of effort to avoid a collision won't be necessarily equal.

In this section, we estimate the value of $\alpha_{r, p}$ for the robot $r$ based on situational factors that describe a given collision situation. This is done by analyzing hundreds of collision avoidance scenarios, particularly the trajectories for each person, which are required to calculate $F\left(\pi_{p}\right)$. This allows us to estimate $\alpha_{r, p}$ by establishing a relationship between 


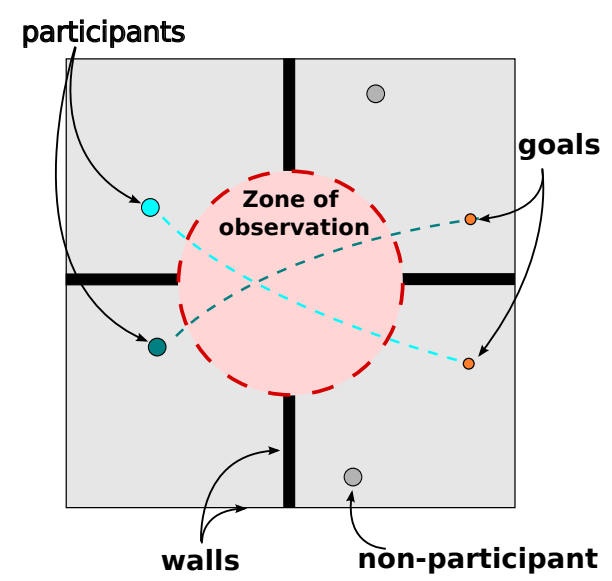

Fig. 2: Experiment area is a square with twelve meters in length. The red circle has six meters of diameter and represents the zone of observation where collision avoidance analysis is made. The circle is virtual. This particular situation represents a scenario with $150^{\circ}$ crossing angle.

the situational factors that describe a given situation and the actual collision avoidance effort distribution.

\section{A. Behavior of people during collision avoidance}

To understand the behavior of people when avoiding collisions among each other, we first designed an experiment involving interactions between two walkers having crossing trajectories. Participants volunteered to perform the experiment and our study conforms the declaration of Helsinki. The experiments were composed of over 450 runs divided near equally into five different crossing angles: $30^{\circ}, 60^{\circ}$, $90^{\circ}, 120^{\circ}$ and $150^{\circ}$. Several filters were used into the data to remove data with too much noise and situations without collision avoidance behavior, after which 202 runs remained for analysis.

The area designed for the experiment is equal to a square with length twelve. As can be seen in Fig. 2, there are occluding walls each with three meters in length at the middle of each square side that are oriented towards its center. The walls are meant to separate participants in such way that they are not able to see all other participants at the same time. This allows walkers to reach a comfort speed and a stable state before interacting with the other walker. Participants were told to reach the opposite side of the experiment area at comfort speed and were informed that they will interact with another walker.

The observation zone in which we concentrate our attention is a circular region with three meters radius in the center of the square. This region is meant to provide sufficient space so that subjects can adapt their speed and orientation during collision avoidance.

Each run of a collision avoidance scenario involves four participants, however only two at a time will ever enter the observation zone at each run. This is necessary as in some crossing scenarios a person may see another one as soon as they are into their initial positions, as such, it is

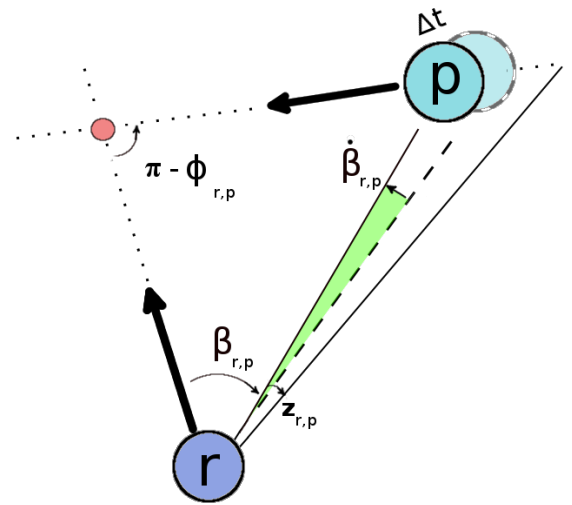

Fig. 3: Collision situation between $r$ and $p$, where crossing angle $\phi$, bearing angle $\beta$ and its derivative $\dot{\beta}$ are shown. The $z$ is calculated based on bearing and crossing angle. Also note that $z_{r, p}=-z_{p, r}$. Arrows indicate a velocity vector.

necessary to add some uncertainty to avoid possibly altering their behavior. The non-participants, as can be see in Fig. 2, won't enter the observation zone and thus do not participate in the collision avoidance.

The synchronization of the participants initial position and start time is done automatically in order to allow for several different collision situations.

\section{B. Roles during collision avoidance}

The information provided in the experimental evaluation of human behavior, detailed in Sec. IV-A, and in accordance to the work of [4], two main roles depending on the crossing order can be identified. Participants are able to identify their future crossing order early in the interaction since few inversions occur within the interaction [4], [7]. This can be done using the optic variable named bearing angle $\beta$ and its derivative, both variables are shown in Fig. 3. The rate of change of a given $\beta$ (its derivative) can be a strong indicator of potential collision. This was established in [16], where a rigorous study of the role of $\beta$ and its derivative $\dot{\beta}$ was made. The derivative of the bearing angle also determines the crossing order, which represents two main roles during collision avoidance: who crosses first and who crosses last. As such, two important conclusions have been reached by analyzing $\beta$ and its derivative during a situation involving a potential collision [16]:

- when $\beta_{r, p}$ is diverging over time i.e. going away from the center of the field view, the agent $r$ is crossing first;

- conversely when $\beta_{r, p}$ is converging over time i.e. going towards the center of the field of view, the agent $r$ is crossing last.

The role of crossing order in a collision situation has been subject of much research. In particular, in [4] for the case of $90^{\circ}$ crossing angle it was shown that the person who is crossing first in average contributes less to avoid a collision than who crosses last. 


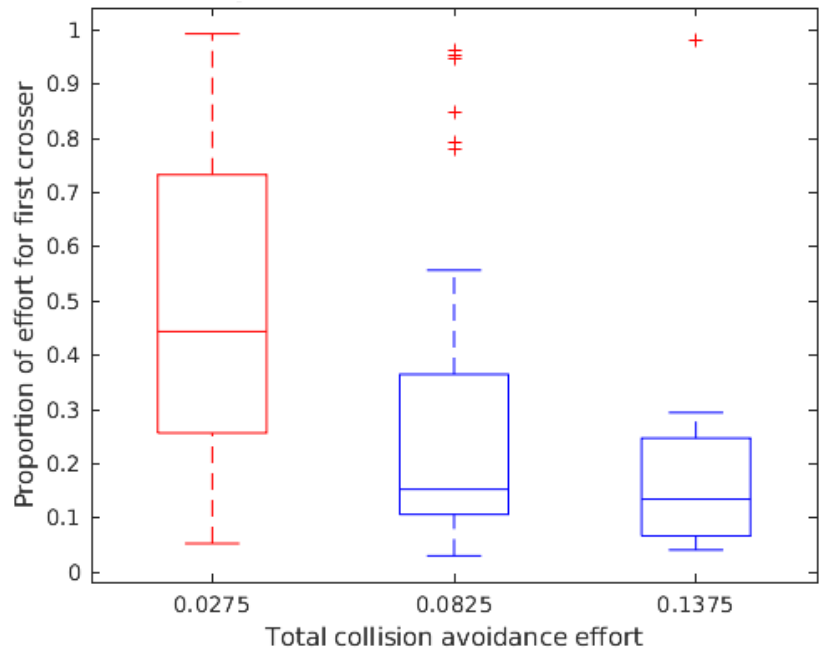

Fig. 4: Proportion of effort that was done by the person who crosses first with respect to total effort required to avoid a collision. In situations that require higher total collision avoidance effort its distribution shift the majority of the effort towards the last crosser. Boxplots in blue indicate that there is a significant difference between the contribution of the first crosser and last crosser ( $\mathrm{p}$-value $<0.05$ ) and in red indicate no significant difference ( $\mathrm{p}$-value $>0.05)$. Values are considered outliers when outside $99.3 \%$ coverage.

\section{Variability in effort distribution}

Let $\pi_{\text {first }}$ and $\pi_{\text {last }}$ be, respectively, the first and last crosser trajectories. Based on the analysis of the whole dataset with people in pairwise interaction to avoid a collision, that was done for each of these roles for all crossing angles, paired ttests show that the last crosser contributed more to collision avoidance task when the combined total, that is $F\left(\pi_{\text {first }}\right)+$ $F\left(\pi_{\text {last }}\right)$, is between 0.55 and 0.165 (p-value $<0.05$ ). There was no significant difference in the low effort condition in the interval between 0 and 0.55 . Thus, whenever a relatively small amount of effort is necessary to avoid a collision the distribution of effort varies without bound between both people, as shown in Fig. 4.

Although a clear reason for this behavior is not apparent, one possible explanation is that crossing order is less relevant in smaller crossing angles, such as $60^{\circ}$, which in our experiments required, in average, less collision avoidance effort. This can be seen in Fig. 5, where the difference in effort distribution between the first and last crosser is comparatively small in comparison to other crossing angles.

\section{Avoiding collision in non-trivial cases}

The proportion of collision avoidance effort required for the person crossing last increases in correlation with the total amount of effort in a given collision avoidance scenario, as was shown in Fig. 4. Thus it is necessary to determine what are the conditions that engender higher or smaller effort in a given collision avoidance scenario.

To explain the effort of a given collision scenario we model it based on four variables: crossing angle $\phi$, difference in speed, deviation from baseline bearing angle $z_{r, p}=\left|\beta_{r, p}\right|-$ $\frac{\phi_{r, p}}{2}$ and time to collision; the time to collision represents the amount of time in which a collision will happen in case no change in the velocity vector is made. Some of these variables can be visualized in Fig. 3 .

Both the difference in people speed and $z$ are calculated in relation to the first crosser. Thus, for each crossing angle we fit the data to a Generalized Linear Model (GLM), with a Binomial distribution and a Logit link function. The GLM was chosen, instead of a linear regression, as it allows for constant change in predictors (situational factors) to be able to cause non-constant change in response. This is important as the increase in total collision avoidance effort causes a non-constant increase in the proportion of effort done by the last crosser.

A GLM model was fitted to the data of the first and of the last crosser $(p$-value $<0.05)$. Do note that these $p$-values are valid only within the region where effort is bigger than 0.035 , given that the spread of effort distribution when absolute collision avoidance effort is small would be too high to allow for reliable prediction.

An example of effort difference between first and last crosser can be seen in Fig. 5, where the evaluated model indicates that, in average, the upper bound for effort invested by the first crosser remains nearly constant while the last crosser effort increases unbounded as the collision avoidance problem becomes more difficult. The cases of highest effort happens whenever the current crossing order is not respected e.g. one agent yields when it should pass first, as can be seen in the lower left of each individual surface in Fig. 5.

Do note that, as stated in Sec. IV-C, in cases which require relatively small amounts of total effort the spread of the effort distribution is high, as such, the effort distribution in these cases is not consistent with the values predicted by our generalized linear model.

\section{E. Discussion}

The manner in which people share effort is consistent over situations with high total collision avoidance effort. Our model allows a robot to evaluate situational factors in order to estimate how effort is distributed among roles. The focus on these factors is inspired on recent works which highlight the importance of situational factors (instead of personal characteristics) in explaining collision avoidance behavior [7]. Although this model can be applied to multiple people by considering each possible pair-wise interaction separately, this could generate unrealistic behavior when dealing with groups of people.

There are other factors that can affect the effort distribution which were not accounted for in this work. For instance, the gender of a person may affect both the degree of collaboration [17], the size of a person's personal space and also group organization [18], which may impact overall behavior. Moreover, a person may decide to yield to a child or an older person even if situational factors indicate that passing first is the expected behavior, in what can be called a polite behavior. 


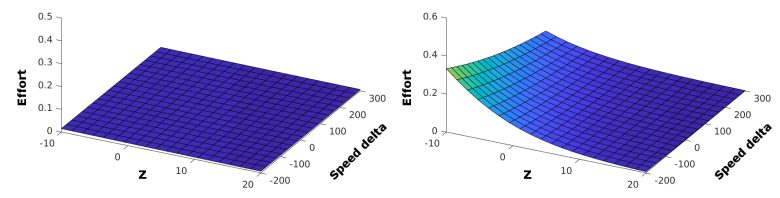

(a) First (left) and last crosser (right). Crossing angle $60^{\circ}$.

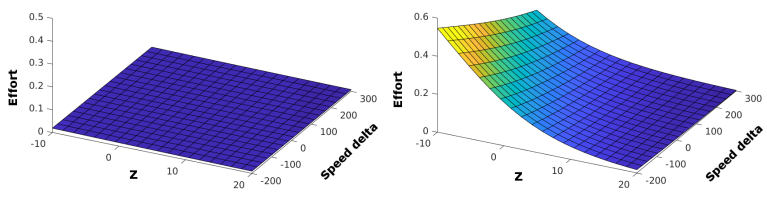

(b) First (left) and last crosser (right). Crossing angle $120^{\circ}$.

Fig. 5: Collision avoidance effort surface, given time to collision of three seconds, for first and last crosser for two crossing angles.

\section{Experiments in ROS using RVO}

The simulated experiments were performed with two main goals in consideration. One is to evaluate if the chosen situational factors are consistent to explain common collision scenarios, while the other helps to validate if the generated effort distribution is consistent with observed data.

\section{A. Finding effort distribution in terms of RVO}

Consider a situation where two agents $q$ and $p$, with desired velocities $v_{q}^{\text {des }}$ and $v_{p}^{\text {des }}$ need to avoid a collision with each other. In this case, RVO finds the smallest change in the relative velocity $\left(v_{q}^{\mathrm{des}}-v_{p}^{\mathrm{des}}\right)$ that avoids a collision between them [19], this is given by $\vec{u}$ as

$$
\vec{u}=\left(\underset{\vec{v} \in \partial \mathrm{VO}_{q \mid p}^{\tau}}{\arg \min }\left\|\vec{v}-\left(\vec{v}_{q}^{\mathrm{des}}-\vec{v}_{p}^{\mathrm{des}}\right)\right\|\right)-\left(\vec{v}_{q}^{\mathrm{des}}-\vec{v}_{p}^{\mathrm{des}}\right)
$$

where $\partial \mathrm{VO}_{q \mid p}^{\tau}$ is the set of changes in relative velocity that are in the threshold between collision and no collision - the minimum change in relative velocity that avoids a collision.

Commonly, RVO shares the change in relative velocity equally between the two agents [19], that is, $\frac{1}{2} \vec{u}$ for each. However, when an agent represents a model of a person's behavior this is not always the correct approach. As such, our work tackles the problem of mapping how much an agent should contribute to avoid a collision into $\alpha$, that is used instead of the equal share. To that end, given the effort defined in Eq. 4, each agent goal and situational factors of all agents, calculating the $k$ that translates to the desired effort distribution in terms of RVO is trivial.

Another issue is the large spread of effort distribution in small collision avoidance effort situations. Given that RVO requires effort to be reciprocal, it's not evident how to translate this optional cooperative effort into its model. Thus, collision avoidance scenarios that require smaller total collision avoidance effort are set to have equal effort distribution while when total collision avoidance increases the effort distribution is shifted to the actual model-based effort distribution value. Thus, let $\mathrm{EDC}=\alpha_{r, p}$, we represent the desired behavior as

$$
f\left(\pi_{p}, \pi_{q}\right)=\frac{1}{1+e^{-\gamma\left(T\left(\pi_{p}\right)+F\left(\pi_{q}\right)-c\right)}}
$$

$$
\operatorname{RVO}-\operatorname{EDC}\left(\pi_{p}, \pi_{q}\right)=0.5+(\mathrm{EDC}-0.5) f\left(\pi_{p}, \pi_{q}\right)
$$

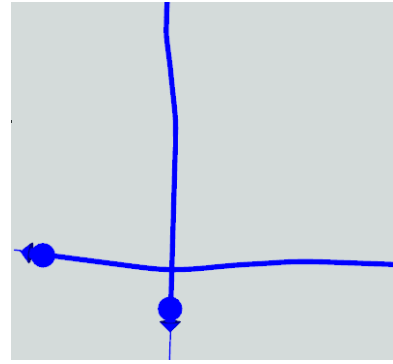

(a) Effort shared between agents. Both change trajectory.

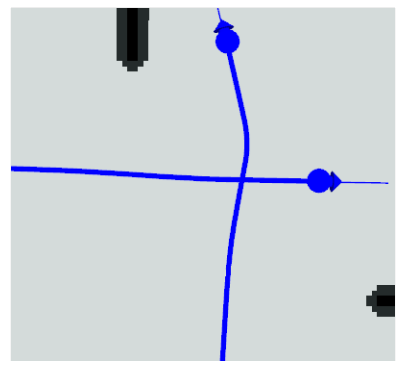

(b) First crosser does all effort to avoid collision.
Fig. 6: Impact of different ways to distribute effort in the generated trajectories.

where $c=0.03$ and $\gamma=260$ are defined based on the empirical data as to allow for smooth transition from equal distribution to using EDC depending on the total collision avoidance effort.

\section{B. Simulated experiments}

In the simulated experiments, the person and the robot are simulated using the same robot model. Furthermore, in order to simulate human speed, the desired speed is set as $1.3 \mathrm{~m} / \mathrm{s}$ and $1.5 \mathrm{~m} / \mathrm{s}$ as maximum speed for the two agents. Using these agents, tests were executed three times for each of these crossing angles: $60^{\circ}, 90^{\circ}, 120^{\circ}$ and $180^{\circ}$. However, in the case of $60^{\circ}$ the examples produced diverged little from standard RVO as the effort distribution in our method approximated equal share.

The first step in the evaluation of our model is to demonstrate the impact of effort distribution in generated trajectories. To that end, the results of the comparison between collaborative and non-collaborative agents are presented in Fig. 6. These results show that the manner in which effort is distributed significantly changes generated trajectories.

The second step of our model requires establishing a comparison between predicted effort distribution in our simulated cases with the baseline obtained from the dataset. For the $90^{\circ}$ experiments, the last crosser tended to change their heading in order to avoid a collision. In our tests, the actual effort distribution was at most within five percentage point difference from the one indicated in our model. This indicates that our implementation is able to translate the RVO-EDC to an appropriate value for a given collision avoidance effort distribution. In contrast, in the $120^{\circ}$ experiments, see Fig. $7 \mathrm{~b}$, agents tended to change their speed (heading is 


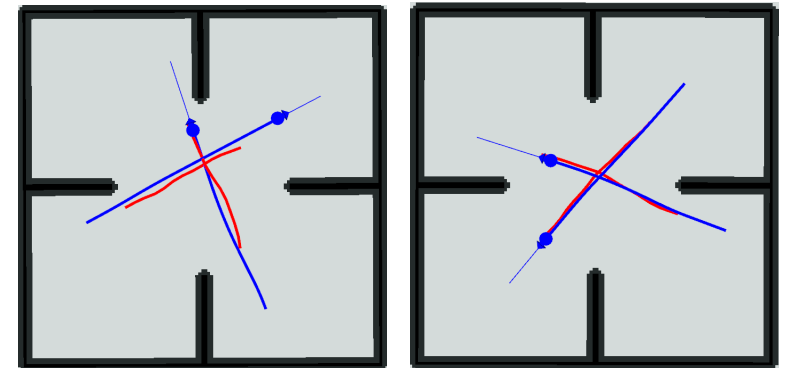

(a) $90^{\circ}$ crossing angle. Hu- (b) $120^{\circ}$ crossing angle. Human $75 \%$, predicted $70 \% \quad$ man $85 \%$, predicted $88 \%$

Fig. 7: Last crosser collision avoidance effort proportion comparison between actual people and simulated data. $\mathrm{Hu}-$ man trajectories are in red, simulated trajectories with our method are in blue.

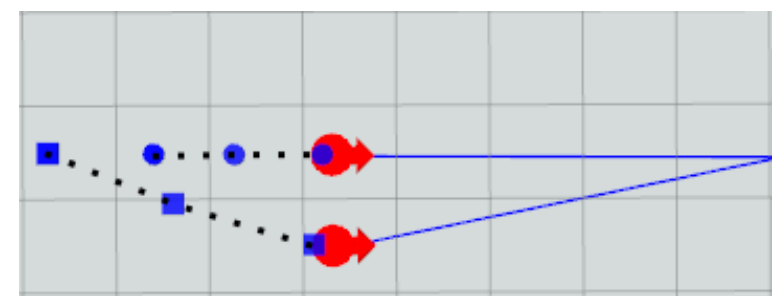

Fig. 8: Behavior when crossing angle is $180^{\circ}$. Agent in front is three times slower and does not contribute in avoiding the collision.

slightly changed), in this case, in our model, the first crosser accelerates less and the last crosser decelerates more.

In order to evaluate if the agent in front does not share collision avoidance effort as expected from our model, in Fig. 8 the $180^{\circ}$ case is tested. In all tests performed, the expected behavior is obtained where the agent in front does not participate in the collision avoidance.

\section{CONCLUSIONS}

People do not always share collision avoidance effort in the same manner, factors such as time to collision and crossing angle affect the proportion each role (first and last crosser) is responsible for. In this work, based on hundreds of collision avoidance situations between people, we evaluated what factors influence the collision avoidance effort distribution in several distinct scenarios. From this, a definition of collision avoidance effort was created and also a model capable of predicting effort distribution. The model shows a clear increase in the relative proportion of effort for the last crosser as the effort increases.

The model was tested in simulated experiments and its result reveals a difference in generated behavior when compared to standard RVO. This difference is more pronounced when a given collision situations requires higher amounts of collision avoidance effort.

It is important to highlight that other factors can influence the manner in which a robot should share effort to others. For instance, a robot can and should yield to a child, given that its awareness of social rules and expectations is not certain when it comes to collision avoidance, even when situational factors would indicate otherwise. As future work, we intend to develop navigation strategies that are better able to represent this non-mandatory cooperation that is present in collision avoidance behavior between people, adding concepts such as politeness in its decision making.

\section{References}

[1] T. Fraichard and H. Asama, "Inevitable collision states-a step towards safer robots?" Advanced Robotics, vol. 18, no. 10, 2004.

[2] F. Lindner and C. Eschenbach, "Towards a formalization of social spaces for socially aware robots," Spatial Information Theory, 2011.

[3] C. Lichtenthaler, T. Lorenzy, and A. Kirsch, "Influence of legibility on perceived safety in a virtual human-robot path crossing task," in IEEE Int. Work. Robot Hum. Interact. Commun., Paris (FR), Sept. 2012.

[4] A. Olivier, A. Marin, A. Crétual, A. Berthoz, and J. Pettré, "Collision avoidance between two walkers: Role-dependent strategies," Gait and Posture, vol. 38, no. 4, 2013.

[5] C. Vassallo, A.-H. Olivier, P. Souères, A. Crétual, O. Stasse, and J. Pettré, "How do walkers behave when crossing the way of a mobile robot that replicates human interaction rules?" Gait \& posture, vol. 60, pp. 188-193, 2018.

[6] D. Carton, W. Olszowy, and D. Wollherr, "Measuring the effectiveness of readability for mobile robot locomotion," International Journal of Social Robotics, 2016.

[7] A. Knorr, L. Willacker, J. Hermsdörfer, S. Glasauer, and M. Krüger, "Influence of person-and situation-specific characteristics on collision avoidance behavior in human locomotion." Journal of experimental psychology: human perception and performance, vol. 42, no. 9, p. 1332, 2016.

[8] D. Claes, D. Hennes, K. Tuyls, and W. Meeussen, "Collision avoidance under bounded localization uncertainty," in IEEE/RSJ Int. Conf. Intell. Robot. Syst. (IROS). IEEE, 2012, pp. 1192-1198.

[9] A. Cosgun, E. Sisbot, and H. Christensen, "Anticipatory robot path planning in human environments," in Robot and Human Interactive Communication, New York (UM), Aug. 2016.

[10] F. Zanlungo, T. Ikeda, and T. Kanda, "Social force model with explicit collision prediction," EPL (Europhysics Letters), 2011.

[11] M. Shiomi, F. Zanlungo, K. Hayashi, and T. Kanda, "Towards a socially acceptable collision avoidance for a mobile robot navigating among pedestrians using a pedestrian model," Int. J. Soc. Robot., vol. 6, no. 3, 2014.

[12] G. Ferrer and A. Sanfeliu, "Proactive kinodynamic planning using the extended social force model and human motion prediction in urban environments," in IEEE Int. Conf. on Intelligent Robots and Systems (IROS), Chicago (UM), Sept. 2014.

[13] G. Silva and T. Fraichard, "Human robot motion: A shared effort approach," in European Conference on Mobile Robots (ECMR). IEEE, 2017, pp. 1-6.

[14] M. Gérin-Lajoie, C. L. Richards, and B. J. McFadyen, "The negotiation of stationary and moving obstructions during walking: anticipatory locomotor adaptations and preservation of personal space," Motor control, vol. 9, no. 3, pp. 242-269, 2005.

[15] M. Zarrugh, F. Todd, and H. Ralston, "Optimization of energy expenditure during level walking," European journal of applied physiology and occupational physiology, vol. 33, no. 4, pp. 293-306, 1974.

[16] J. Cutting, P. Vishton, and P. Braren, "How we avoid collisions with stationary and moving obstacles," American Psychological Association, vol. 102, no. 4, 1995 .

[17] B. Van Basten, S. Jansen, and I. Karamouzas, "Exploiting motion capture to enhance avoidance behaviour in games," in International Workshop on Motion in Games. Springer, 2009, pp. 29-40.

[18] M. Costa, "Interpersonal distances in group walking," Journal of Nonverbal Behavior, vol. 34, no. 1, pp. 15-26, 2010.

[19] J. van den Berg, S. Guy, M. Lin, and D. Manocha, Reciprocal n-Body Collision Avoidance. Berlin, Heidelberg: Springer Berlin Heidelberg, 2011. 